\title{
Improvements in the realization of the triple point of water in metallic sealed cells at LNE-Cnam
}

\author{
Carolina Cappella ${ }^{1, \star}$, Fernando Sparasci ${ }^{1}$, Laurent Pitre ${ }^{1}$, Bruno Buée ${ }^{1}$, and Ahmed El Matarawy ${ }^{2}$ \\ 1 LNE-Cnam, 61, rue du Landy, 93210 La Plaine Saint Denis, France \\ 2 Thermal Metrology Laboratory, NIS, Egypt
}

Received: 30 July 2015 / Accepted: 31 October 2015

\begin{abstract}
Since 2008, LNE-Cnam has been working on the development of metallic sealed cells for the water triple point, temperature $273.16 \mathrm{~K}$. The objective is to perform calibrations of capsule-type standard platinum resistance thermometers employing adiabatic calorimetry techniques, in order to get the lowest possible level of measurement uncertainty. After the realization of a preliminary prototype, the full system composed of new cells and an adiabatic calorimeter has been significantly improved. New cells have been manufactured, an ameliorated calorimeter has been built and improved procedures have been set up to clean, fill and seal the cells. Despite a remaining discrepancy of about $0.8 \mathrm{mK}$ between metallic and traditional water triple point cells, the reproducibility of metallic cells has been improved to $0.1 \mathrm{mK}$. This paper describes the new devices developed and the results obtained. This work has been developed within the frame of the European metrology research program (EMRP) joint research project 'SIB10 NOTED'. The EMRP is jointly funded by the EMRP participating countries within EURAMET and the European Union.
\end{abstract}

Keywords: Water triple point, fixed points, adiabatic calorimeter, CSPRT, metallic cell

\section{Introduction}

The triple point of water (TPW, temperature $273.16 \mathrm{~K}$ ) defines the kelvin and is one of the defining fixed points of the International Temperature Scale of 1990 (ITS-90). The TPW is the most critical point in the ITS-90, because it is the reference point in the calibration of Standard Platinum Resistance Thermometers (SPRTs). It is also the connection between the current definition of the kelvin and the future one based on the Boltzmann constant.

The TPW is practically realized by means of sealed borosilicate-glass or fused-quartz cells, filled with highpurity water [1]. Triple point realizations under adiabatic conditions [2] are not possible with this configuration and the melted ice fraction during the TPW cannot be easily determined. Metallic sealed cells appear to be an alternative to glass cells allowing the supply of controlled heat pulses, in order to melt the sample under adiabatic conditions, inside an accurately temperature-controlled calorimeter [3]. Moreover, since copper shows a very good thermal conductivity, metallic sealed cells realized in copper allow fast thermal response time and excellent thermal uniformity, with potentially better uncertainties.

This work presents the improvements in the development of sealed copper cells for the triple point of water, with respect to what was published in reference [2]. New

\footnotetext{
^ Correspondence: maria-carolina.cappella@cnam.fr
}

cells have been manufactured, an ameliorated calorimeter has been built and improved procedures to clean, fill and seal the cells have been set up. Despite a remaining discrepancy of about $0.8 \mathrm{mK}$ between metallic and traditional water triple point cells, the reproducibility and the time-stability of metallic cells have been improved to the level of $0.1 \mathrm{mK}$.

\section{Description of the cell}

The metallic cell designed to realize the TPW is presented in Figure 1.

\subsection{Choice of material}

The whole cell is made with copper. From the thermal point of view, the high thermal conductivity of copper ensures a small resistance between the sample of water and the external wall of the cell, where thermometers are located. This reduces the risk of offsets in temperature measurements and allows reliable evaluations of the timeconstant and recovery-time of the cell. From the chemical point of view, cuprous ions can be slightly soluble in water [4], so the impurity effect due to copper contamination cannot be neglected. One way to avoid this impurity 

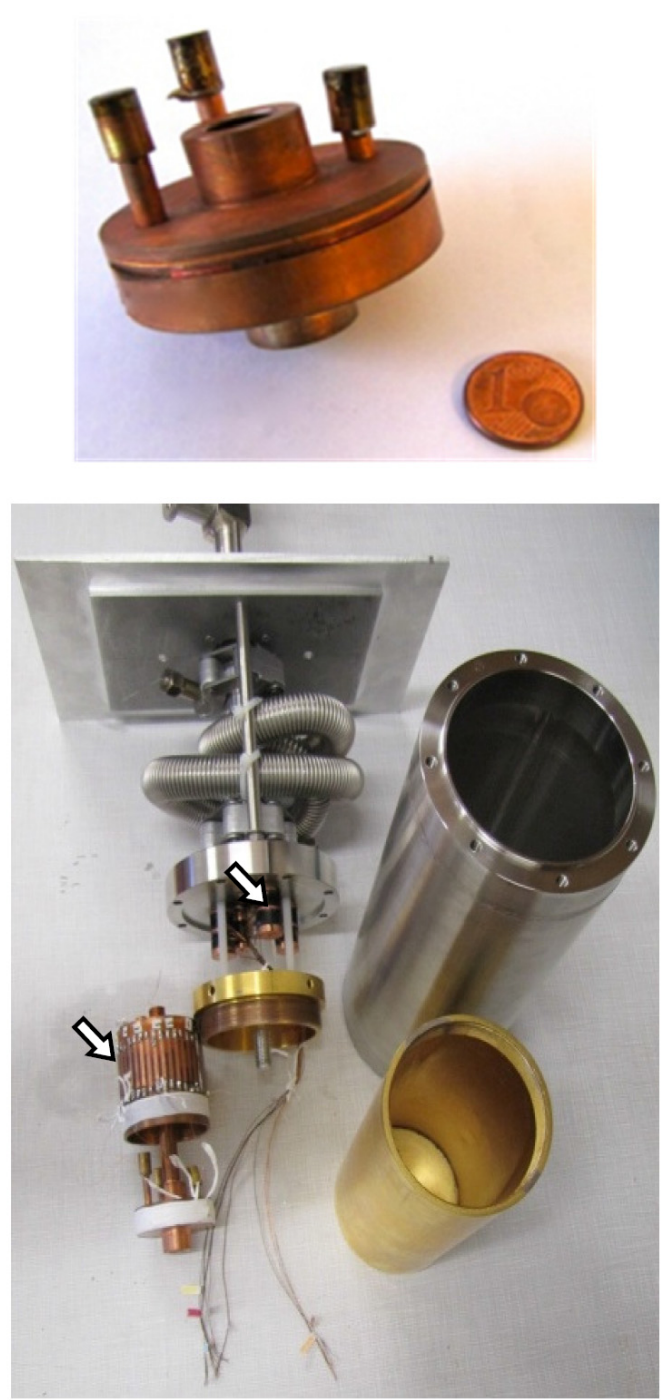

Fig. 1. The copper TPW cell and the adiabatic calorimeter. The two arrows show the thermal anchoring of the electrical wires.

source is to create an oxide layer in the copper cell, in order to separate the non-oxide copper layer from the pure water [2].

\subsection{Geometry}

The geometry of the cell is chosen in order to allow an easy assembling with a copper block, where it is possible to hold up to eight capsule-type thermometers. The interest is to perform calibrations of several sensors in a single run.

The cell is cylindrical, with diameter $40 \mathrm{~mm}$ and height $8 \mathrm{~mm}$, and has threaded ends for the assembly with the thermometer block. Three filling tubes of $2 \mathrm{~mm}$ of diameter allow a simple filling and cleaning procedure, which is realized with a continuous water flow, to remove impurities and realize the oxide layer inside the cell. They enable also leak tests or doping experiments with determined amount of impurities.
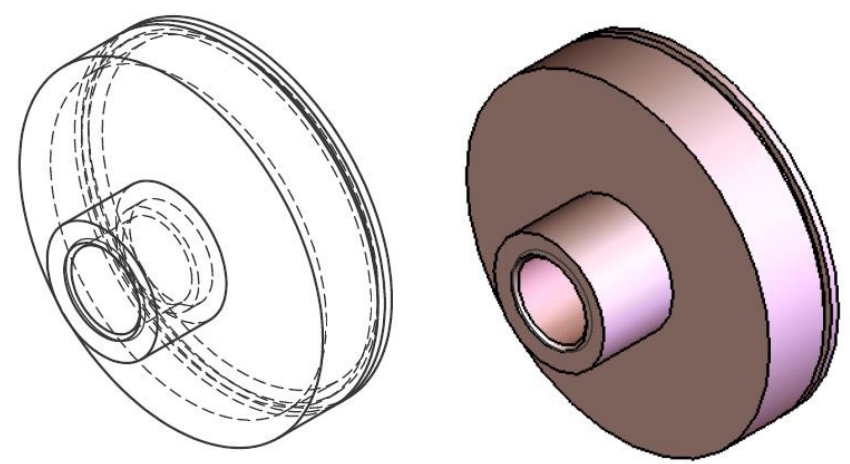

(a)

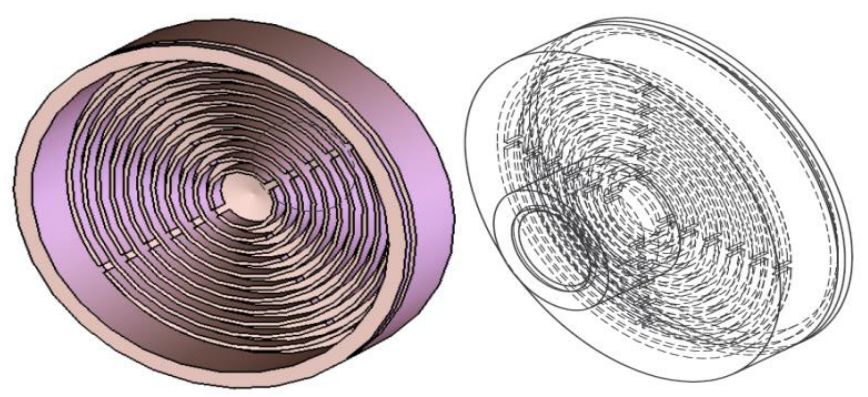

(b)

Fig. 2. Designs of the inside of the copper TPW cell: (a) cambered smooth cell (CSC) and (b) flat toothed cell (FTC).

Two different layouts have been adopted for the inside of the cell: "cambered smooth cell (CSC)" and "flat toothed cell (FTC)" (Fig. 2). With respect to the FTC design, CSC cells offer a wider contact surface between the water and the metal. They reproduce the design of the cryogenic "MULTICELLS" developed in the past years by the laboratory [5]. The two different layouts were adopted to check whether the structure affects the thermal characteristics of the cell.

\subsection{Cleaning and filling procedure}

The cleaning procedure consists in flushing the cell with a continuous flow of ultra-pure water during two weeks, in order to remove impurities and create an oxide layer inside. To ensure water purity, the water coming from a reverse osmosis plant is bi-distilled and directly supplied to the filling system, avoiding contamination due to storage. The filling system and the cleaning procedure are described in reference [6]. After two-weeks flushing, the ultra-pure water is outgassed under vacuum, to prevent air contamination and the dissolution of carbon dioxide, which is one of the atmospheric gases with the highest absorption coefficient [2]. A first outgassing is performed by pumping over the liquid water inside a first flask. Then, the outgassed water is evaporated under vacuum and collected into a second flask, kept under vacuum as well, where the outgassing is repeated. Finally the water is vaporized from the second flask and collected into the cell, which is also under vacuum. A borosilicate-glass 


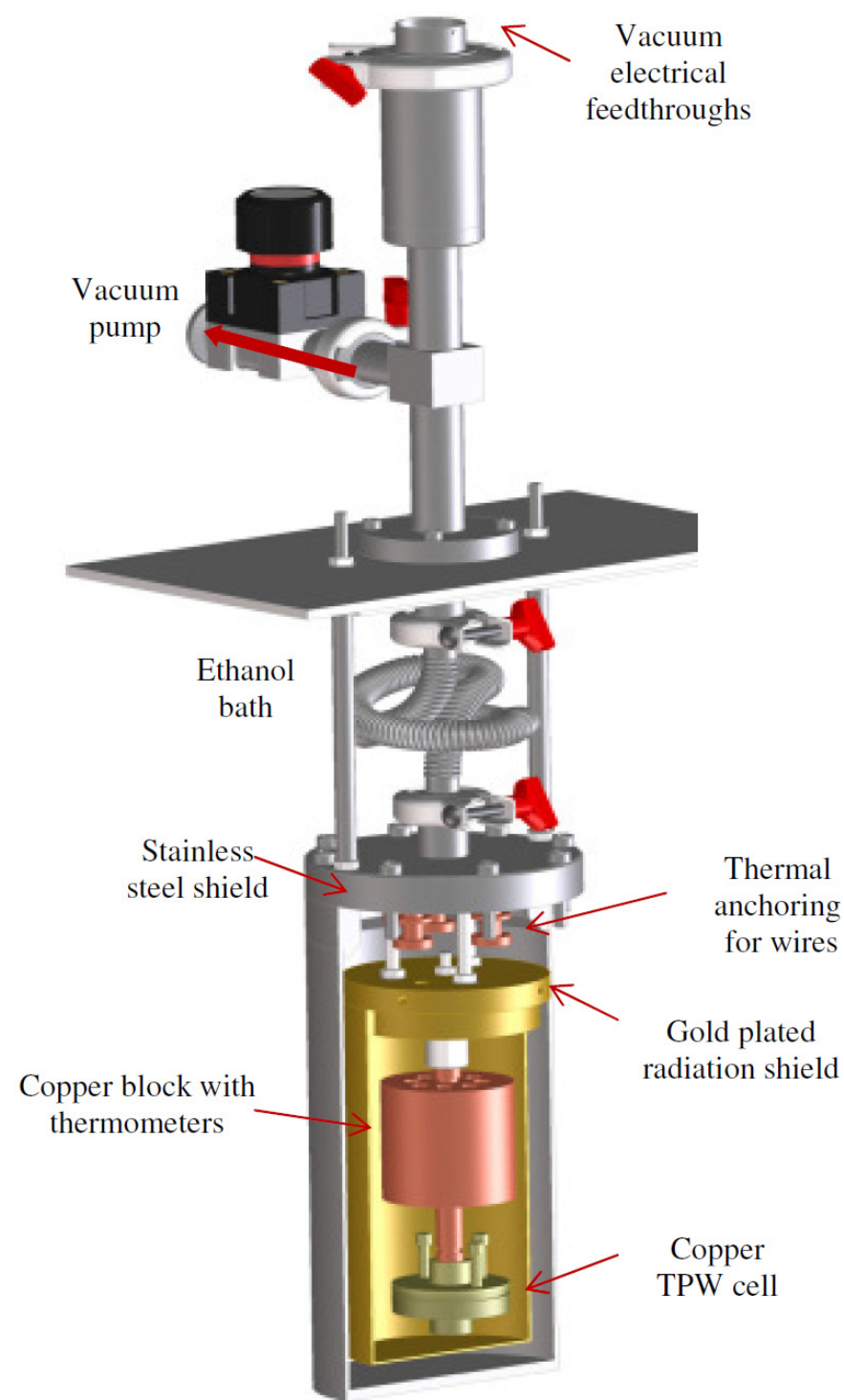

Fig. 3. Design of the adiabatic calorimeter.

graduated pipette, connected to the input of the cell, allows to roughly estimate the amount of liquid water in the cell. At the end, the inlet and outlet tubes are permanently sealed by pinching-off.

The maximum water content allowed in the TPW cell is about $9 \mathrm{~g}$. We decided to fill the TPW cell with around $4 \mathrm{~g}$ of water, to obtain cells with a heat of fusion close to $1340 \mathrm{~J}$.

An improved new filling bench is under construction. Unlike the system described in reference [6], in the new bench all the silicon parts will be removed. All the tubes and stoppers will be in borosilicate glass, to improve outgassing capability and enhance water purity.

\subsection{Description of the adiabatic calorimeter}

The design of the adiabatic calorimeter is presented in Figure 3. The adiabatic calorimeter is composed of a cylindrical, stainless-steel vacuum can, with inside a goldplated, copper thermal shield, to avoid the effect of the radiation from the outside. The thermal shield is temperature-controlled by means of a $50 \Omega$ heater and a $100 \Omega$ platinum thermometer (Pt-100), both glued on it. Vacuum inside is of about $10^{-2} \mathrm{~Pa}$, and the calorimeter is placed in a temperature-controlled ethanol bath Fluke model 7381, with a stability of $5 \mathrm{mK}$.

Inside the thermal shield, there are the copper TPW cell and the copper block for thermometers. The copper block is cylindrically symmetric and has eight wells in the annular region, where Capsule-type Standard Platinum Resistance Thermometers (CSPRTs) to be calibrated can be located. In order to increase the thermal link with the copper block, the CSPRTs are coated with vacuum grease.

All the electrical leads are thermally anchored to the container. The first anchoring point is realized on the vacuum flange. Here the wires are wound around some copper coils screwed into the flange, and are dipped in a lowtemperature epoxy coating. The second anchoring is realized by means of copper strips deposited on a polyimide layer pasted over the copper block for thermometers. The picture in Figure 1 shows the location of the anchoring points inside the cryostat.

A Pt-100 thermometer and a $150 \Omega$ heater, both glued onto the radiation shield, are used to monitor and control its temperature, via an $8^{1 / 2}$ digit digital multimeter, fourwire connected to the Pt-100.

A heater of $50 \Omega$ glued onto the cell is used to supply controlled heat pulses during the realization of the phase transition (plateau).

Instrumental control and data acquisition functions are operated with a computer using a software developed in the laboratory for regular calibrations and conformable with the standard ISO 17025.

\section{Results and discussions}

\subsection{Measuring instruments}

One CSPRT, Goodrich model 162D, serial number 5337, was used to carry out measurements. It was preliminarily calibrated according to the ITS-90 definition, at the TPW and the melting point of Gallium, with calibration uncertainties of $0.12 \mathrm{mK}$ and $0.19 \mathrm{mK}$ respectively. With these uncertainties, the CSPRT was considered reliable for this study. A calibrated standard reference resistor of $100 \Omega$ (Tinsley, model 5685A, serial number 8139/18), maintained in a temperature-controlled oil bath at $25{ }^{\circ} \mathrm{C}$, was conjugated with an Automatic Systems Laboratories, model F18 AC resistance bridge, to measure the resistance ratio of the thermometer with respect to the standard reference resistor. Experimental data were corrected by thermometer self-heating effect, performing measurements at $1 \mathrm{~mA}$ and $\sqrt{2} \mathrm{~mA}$. Data were extrapolated to $0 \mathrm{~mA}$.

Two cells were measured, one with the CSC internal layout, and one with FTC design. 


\subsection{Calorimeter characterization}

In order to estimate the measurement accuracy of the adiabatic calorimeter, a characterization similar to that presented in [7] was realized, measuring the heat capacity $C_{\mathrm{C}}$ of the cell and the thermal resistance $R_{\mathrm{S}}$ between the cell and the adiabatic shield, at temperatures close to the TPW.

The cell heat capacity was determined by measuring the amount of heat energy required to increase the temperature of the cell by a known amount. The energy supplied to the cell was determined from the electrical power applied to the heater around the cell:

$$
C \mathrm{c}=Q / \Delta T=P t / \Delta T
$$

where, $C c\left(\mathrm{~J} \mathrm{~K}^{-1}\right), Q(\mathrm{~J})$ and $P t(\mathrm{~W})$ are the heat capacity, the amount of heat and the electrical power, respectively. From these measurements, we obtained a heat capacity $C c=328.13 \mathrm{~J} \mathrm{~K}^{-1}$.

The thermal resistance was determined monitoring both the temperature drifts of the cell and the shield after a sudden change in the temperature of the shield.

$$
R s=\frac{(T s-T c)}{(C c(d T c / d t))}
$$

where, $R_{S}\left(\mathrm{~K} \mathrm{~W}^{-1}\right), T_{S}(\mathrm{~K}), T_{C}(\mathrm{~K})$ and $d T_{C} / d t\left(\mathrm{~K} \mathrm{~s}^{-1}\right)$ are the thermal resistance, the temperature of the shield, the temperature of the cell and the temperature drift of the cell, respectively. We obtained $R_{S}=6285.60 \mathrm{~K} \mathrm{~W}^{-1}$.

\subsection{Recovery time}

The dynamic behaviour of the cell determines the thermal recovery after a heat pulse, when the phase transition is ongoing. Considering that $\Delta T$ is the temperature difference between the maximum temperature after a heat pulse $T_{\text {pulse }}$ and the plateau temperature $T_{\text {Plateau }}$

$$
\Delta T=T_{\text {pulse }}-T_{\text {plateau }}
$$

the recovery time is determined as the time-interval in which the temperature decreases from $T_{\text {pulse }}$ to $T_{\text {plateau }}+$ $0.05 \Delta T$.

Triple point measurements were realized applying a series of controlled heat pulses through the heater mounted on the metallic TPW cell. Fifteen heat pulses of $89 \mathrm{~J}$ were applied on each plateau realization, providing a total energy of $1340 \mathrm{~J}$. Overheating after each heat pulse went from $10 \mathrm{mK}$ at the beginning of the melting, up to $20 \mathrm{mK}$ at the end of the melting plateau for the two cells tested. Plateau duration was on the order of $83 \mathrm{~h}$, with $5 \mathrm{~h}$ of relaxation time between pulses. The cells showed an average time decay close to $8 \mathrm{~min}$.

Figure 4 shows the recovery time as a function of the melted fraction $F$.

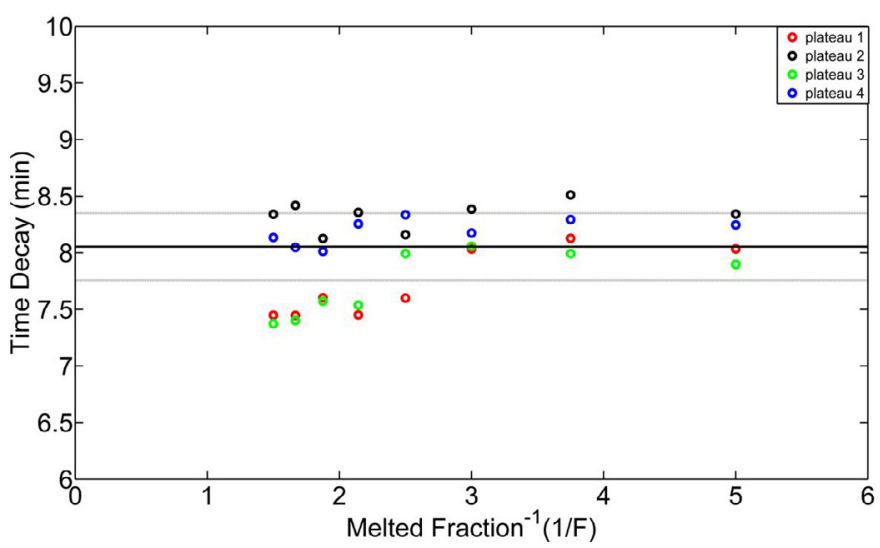

Fig. 4. Evaluation of the recovery time for different $F$ values.

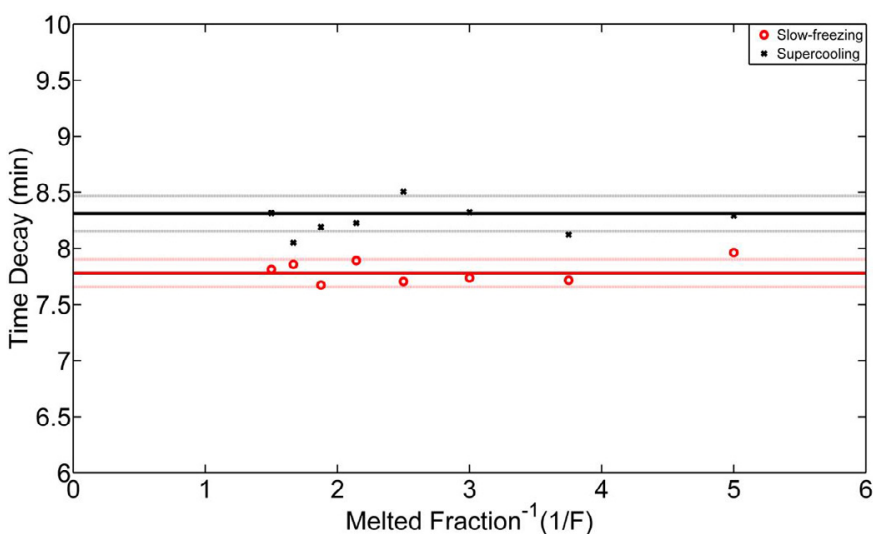

Fig. 5. The dependence of the melting temperature and the time recovery conditions in the obtaining of the solid phase.

\subsection{Temperature control of the system}

\subsubsection{Supercooling vs. slow-freezing}

Two techniques to solidify the water sample inside the cell were tested. The first was a quick freeze at the supercooling temperature, inducing ice crystal formation around impurities, which creates regions in the solid sample with different melting temperatures [8]. The second technique was a slow-freeze, performed after a preliminary incomplete melting at $90 \%$, where freezing occurs slowly around the residual ice, allowing a migration of the impurities in the remaining liquid.

We evaluated the dependence of the melting temperature and recovery time from the freezing conditions.

We did not notice a clear correlation between the extrapolated temperatures at $F=100 \%$ and the freezing technique, as shown in Figure 5.

We observed instead that the recovery time is slightly broadened when a supercooling is performed, as can be seen in Figure 5. We suppose an effect of the ice crystal defects, because the quality of the ice crystal structure is affected by the spatial distribution of the impurities in the cell [8]. 


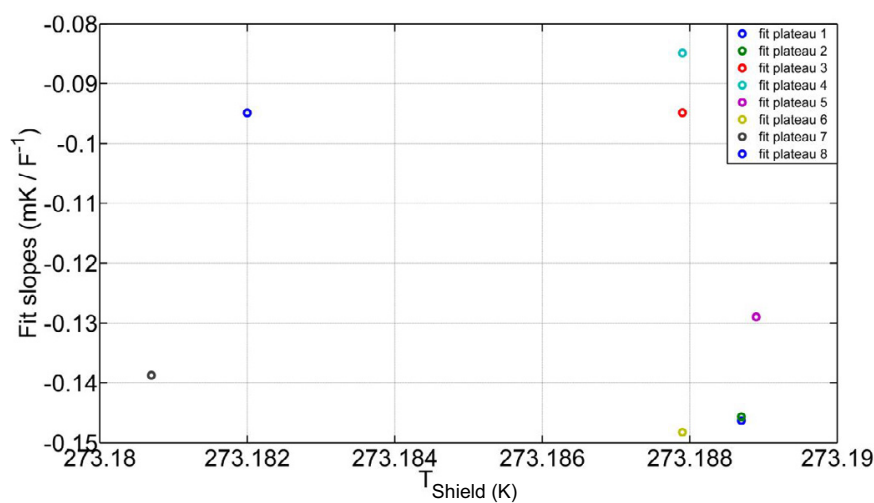

(a)

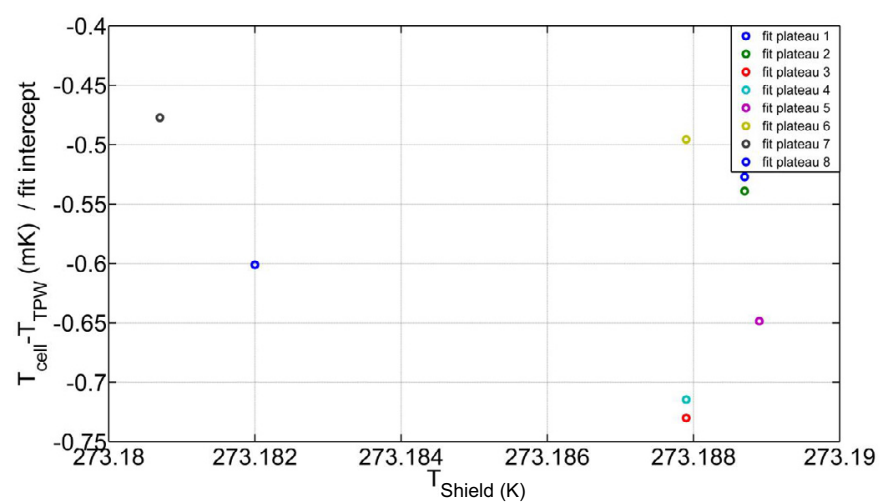

(b)

Fig. 6. Influence of the shield temperature on (a) the slope and (b) the intercept.

\subsubsection{Effect of heat fluxes}

Measurements on the CSC cell were carried out changing either the shield temperature, or the bath temperature during the plateaus. We expected a correlation between these changes and the extrapolated temperatures at $F=100 \%$, or with the slopes of the extrapolated plateaus. However, as we can see in Figures 6 and 7, there is no evidence of such a correlation and, up to now, we did not find a clear explanation for the scattering of the points shown in these figures.

\subsection{Reproducibility of the TPW}

Six plateaus were realized with the CSC cell, and five with the FTC cell, over a period of 18 months. The CSPRT resistance was measured at each melted fraction $F$ after each heat pulse. Results were extrapolated from a first-order polynomial fit at the liquidus point, from measurements at $F$ between $25 \%$ and $75 \%$ (Fig. 8 ).

The CSC cell showed an average extrapolated temperature about $0.8 \pm 0.1 \mathrm{mK}$ below the TPW temperature (realized with traditional glass cells). The FTC temperature was $1.2 \pm 0.06 \mathrm{mK}$ below the TPW. The uncertainties given here represent only the reproducibility of the plateau, and are calculated from the scattering of liquidus

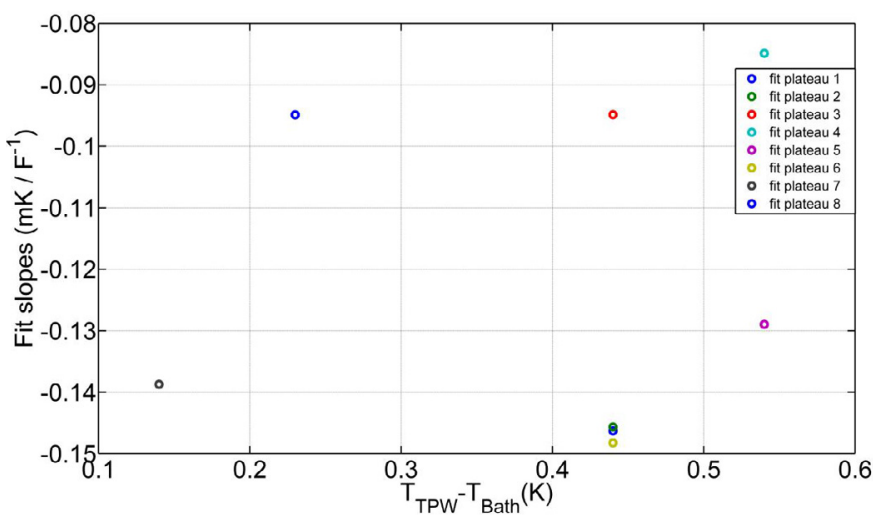

(a)

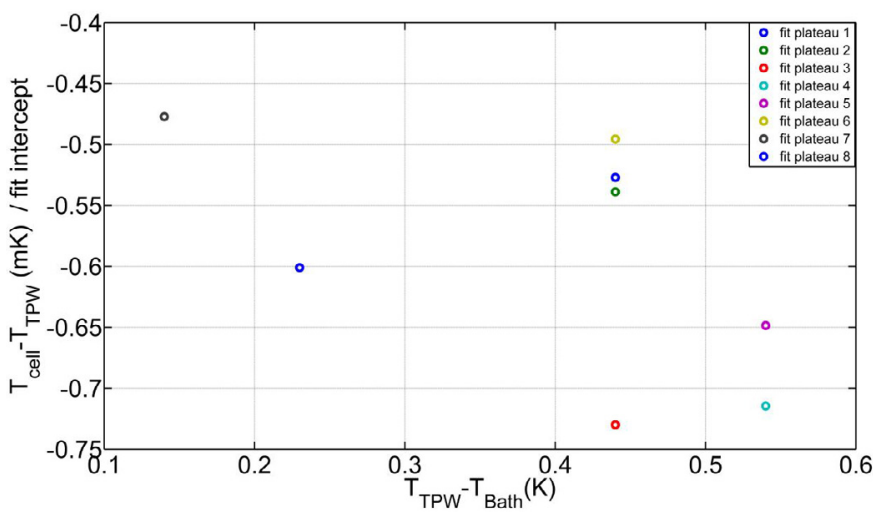

(b)

Fig. 7. Influence of the bath temperature on (a) the slope and (b) the intercept.

point extrapolations. They are not representative of a full uncertainty budget.

We make two hypotheses to explain the difference of $0.4 \mathrm{mK}$ between the two cells. The first is that the impurity content is different in the water samples. With the current filling system we are unable to collect water samples for chemical analyses. Therefore we cannot fully assess that the filling process runs identically on each cell. The future filling system, currently under construction, will ensure a more reproducible filling process and will allow water sample collection for chemical analysis. The second hypothesis is that the layout affects the thermal behaviour of the cells. We will perform further investigations but we consider this assumption less probable, as the high thermal resistance $R_{S}$ (see Sect. 3.2) and the thermal anchoring of the electrical leads (see Sect. 2.4) should considerably limit the effect of the parasitic heat flux.

The main improvement in the new cells presented in this paper concerns the time-stability of the temperature of the phase transition. The cells presented in reference [2], were affected by a drift in the phase transition temperature of $0.42 \mathrm{mK}$ year $^{-1}$, symptom of a probable progressive pollution of the water sample inside the cell.

Here, no time-related temperature drift was observed in both CSC and FTC cells, over 18 months of experiments. We consider that the changes in the cleaning and 


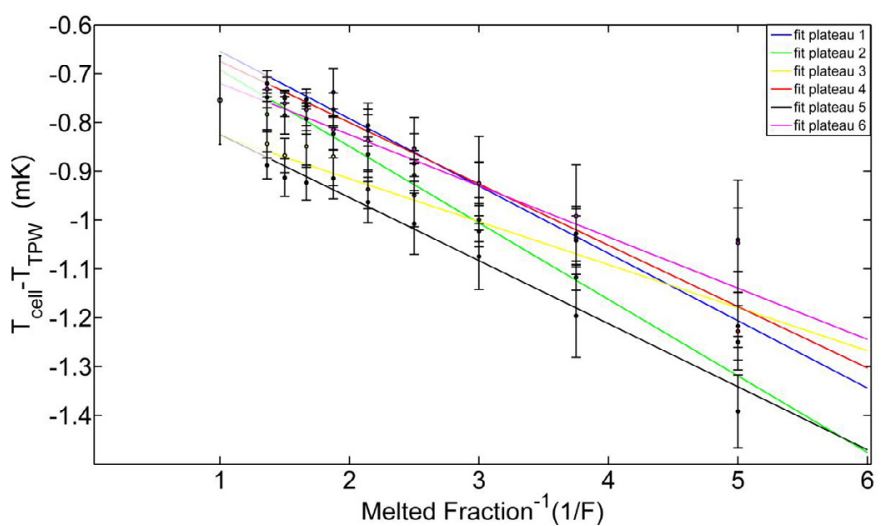

(a)

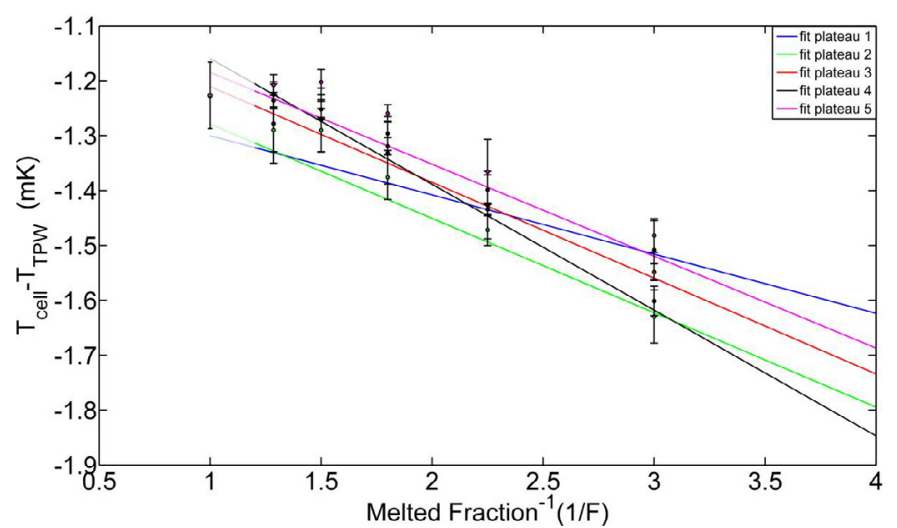

(b)

Fig. 8. Melting plateaus realized with adiabatic calorimetry techniques, the dashed lines show the extrapolation of a fit to $100 \%$ of $F($ a) CSC (b) FTC.

filling procedures have considerably improved the quality of the copper-oxide layer inside the cells, which reduces the risk of progressive contamination of the water sample.

\section{Conclusion}

The results obtained on an improved device employing adiabatic calorimetry techniques and metallic TPW cells for the calibration of CSPRT have been presented and commented.

Further investigations are necessary to explain the influence of the chemical impurities of the system in the results obtained. We plan to realize new cells with an improved filling facility, where the content of impurities in the water sample should be substantially reduced with respect to the current system. We will carry out also additional tests with the cells presented in this paper, by heating them above the ambient temperature, in order to accelerate any possible chemical pollutions and observe their effect on the phase transition.

We consider that the results achieved with this experiment, consisting in a reproducibility of the phase transition temperature below $0.1 \mathrm{mK}$ and in a time-stability over several months are strongly encouraging. We will pursue our efforts in the development of metallic cells for the triple point of water.

Acknowledgements. This work has been developed within the frame of the European Metrology Research Program (EMRP) joint research project 'NOTED'. The EMRP is jointly funded by the EMRP participating countries within EURAMET and the European Union.

\section{References}

1. A. Peruzzi, M. Dobre, J. van Geel, A. Uytun, M. Kalemci, E. Uysal, G. Strouse, Y. Nuevo Ordonez, C. Davis, Effect of impurities on water triple-point cells, Int. J. Thermophys. 35, 1084-1096 (2014).

2. F. Sparasci, L. Pitre, Y. Hermier, Realization of the triple point of water in metallic sealed cells at the LNEINM/CNAM: A progress report, Int. J. Thermophys. 29, 825-835 (2008).

3. Y. Hermier, G. Bonnier, in TEMPMEKO ' 87 , 3rd International Symposium on Temperature and Thermal Measurements in Industry and Science, 1987, pp. 33-39.

4. D.A. Palmer, P. Bénézeth, Solubility of copper oxides in water and steam, in 14 th International Conference on the Properties of Water and Steam in Kyoto, 2008, No. I, pp. 491-496.

5. D. Hermier, Y. Pitre, L. Geneville, C. Vergé, A. Bonnier, G. Head, A new generation of multicells for cryogenic fixed points at BNM/INM, in AIP Conf. Proc., 2003, Vol. 684, p. 179.

6. B. Buée, A. Vergé, V. Vidal, E. Georgin, F. Sparasci, Copper passivation procedure for water-filled copper cells for applications in metrology, Phys. Instrum. Detect. arXiv: 1211.7294 (2012).

7. F. Sparasci, L. Pitre, G. Rouillé, J.-P. Thermeau, D. Truong, F. Galet, Y. Hermier, An adiabatic calorimeter for the realization of the ITS-90 in the cryogenic range at the LNE-CNAM, Int. J. Thermophys. 32, 201-214 (2011).

8. L. Wolber, B. Fellmuth, Influence of the freezing and annealing conditions on the realization of cryogenic triple points, Int. J. Thermophys. 29, 82-92 (2007). 